\title{
Incidental Detection of Inguinoscrotal Bladder Hernia with Associated Factors on ${ }^{18} \mathrm{~F}$-fluorodeoxyglucose PET/CT Scans
}

\author{
Gulay Acar ${ }^{1}$ and Ozlem Sahin ${ }^{2}$ \\ ${ }^{1}$ Department of Anatomy, Meram Faculty of Medicine, Necmettin Erbakan University, Meram, Konya, Turkey \\ ${ }^{2}$ Department of Nuclear Medicin, Meram Faculty of Medicine, Necmettin Erbakan University, Meram, Konya, Turkey
}

\begin{abstract}
Objective: To evaluate the associated factors of inguinoscrotal bladder hernia (ISBH), which was detected on ${ }^{18} \mathrm{~F}$-fluorodeoxyglucose positron emission tomography/computed tomography $\left({ }^{18} \mathrm{~F}-\mathrm{FDG} \mathrm{PET} / \mathrm{CT}\right)$ scans.

Study Design: An observational study.

Place and Duration of Study: Department of Nuclear Medicine, Meram Faculty of Medicine, Necmettin Erbakan University, Konya, Turkey, from August 2010 to April 2021.

Methodology: In this study, 60 cases were subclassified in three age-matched groups; 15 patients diagnosed with ISBH in Group Ia, 15 cases with inguinoscrotal herniation (ISH) of bowel in Group Ib, and 30 patients without ISH in Group II. In the reconstructed $\mathrm{PET} / \mathrm{CT}$ images, the prostate volume (PV), abdominal subcutaneous fat (SFV), and visceral fat (VFV) volumes were measured, and body mass index (BMI) was calculated.

Results: There was a statistically significant relationship between subgroups of Group I in the measurements of BMI, PV, SFV, and VFV, which were higher in Group la than Group Ib. The lower SFV/VFV value in Group lb was considered as the most important risk factor in developing ISH of the bowel. A significant statistical difference was found between Group I and II in terms of PV, VFV, and SFV/VFV parameters.

Conclusions: This study reiterated the impact of PV and the parameters representing abdominal fat distribution on ISBH development. PET/CT scan provided valuable information regarding both the hernia contents and the associated factors in which the higher PV had a significant association for the development of ISBH as well as lower SFV/VFV that was more prominent in ISH with bowel.
\end{abstract}

Key Words: Inguinoscrotal bladder hernia, ${ }^{18}$ F FDG PET/CT scan, Subcutaneous fat, Prostate volume, Visceral fat.

How to cite this article: Acar G, Sahin O. Incidental Detection of Inguinoscrotal Bladder Hernia with Associated Factors on ${ }^{18} \mathrm{~F}$ fluorodeoxyglucose PET/CT Scans. J Coll Physicians Surg Pak 2022; 32(03):283-287.

\section{INTRODUCTION}

The imaging characteristics of inguinal hernias that contain bowel, fatty tissue, bladder or ovary can be demonstrated by using ${ }^{18} \mathrm{~F}$ fluorodeoxyglucose positron emission tomography/computed tomography $\left({ }^{18} \mathrm{~F}-\mathrm{FDG} \mathrm{PET} / \mathrm{CT}\right)$. Approximately 20 million patients with a higher incidence in males (male/female, $8 / 1$ ) require a surgical repair due to the risk of incarceration or strangulation. ${ }^{1}$ Inguinoscrotal bladder hernia (ISBH) is a rare (1-4\%) clinical condition presenting with a major male predilection (70\%), and the highest occurrence is between 50 and 70 years of age. An estimated incidence may reach up to $10 \%$ in obese males aged $\geq 50$ years. ${ }^{2,3}$

Correspondence to: Dr. Gulay Acar, Department of Anatomy, Meram Faculty of Medicine, Necmettin Erbakan University, Konya, Turkey

E-mail: gulayzeynep73@gmail.com

Received: August 04, 2021; Revised: November 03, 2021; Accepted: December 27, 2021

DOI: https://doi.org/10.29271/jcpsp.2022.03.283
Most of ISBHs are asymptomatic and usually detected in the right inguinal region during radiologic imaging or herniorrhaphy surgeries. The most common risk factors contributing to the development of ISBH, in descending order, benign prostate hyperplasia (BPH), obesity or other abdominal pathologies increasing intra-abdominal pressure, an acquired weakness of the abdominopelvic and bladder walls in adults. ${ }^{4-6}$ Surgical repair with bladder reduction or partial bladder resection is a major operation that is generally required in ISBH, bladder wall necrosis, and cancer in the herniated bladder. ${ }^{5,7}$

In literature, cystography is the gold standard diagnostic modality, as well as ultrasound, which confirms the diagnosis. Computed tomography (CT), intravenous urography, and magnetic resonance (MR) imaging were reported as the primary preoperative methodology applied for imaging of ISBH to provide essential information for surgical planning. ${ }^{8} \mathrm{PET} / \mathrm{CT}$ imaging allows full body acquisition from the skull base to the midthigh (cranial to caudal) that facilitates incidental diagnosis of asymptomatic inguinal bladder herniation. The visualisation 
pattern of ISBH on PET/CT images may be misinterpreted as metastasis due to increased FDG uptake in the herniated bladder. So, simultaneous interpretation of the PET images with the combined CT data is of utmost importance for differential diagnosis of tumors, preventing misinterpretation as metastasis, and to avoid bladder injury during herniorrhaphy. .,9-11 $^{-11}$

Since ISBH is a potential but uncommon cause of incarceration and obstructive bladder dysfunction, an information on risk factors related to body fat composition and the volume of prostatic tissue are needed. Furthermore, there is no clinical study investigating the diagnostic utility of ${ }^{18} \mathrm{~F}$-FDG PET/CT in detecting ISBH and the impacts of the volumes of prostatic tissue, subcutaneous and visceral fat on the development of an inguinoscrotal hernia (ISH).

The objective of the present study was to demonstrate the association of major risk factors for ISH with bladder or bowel.

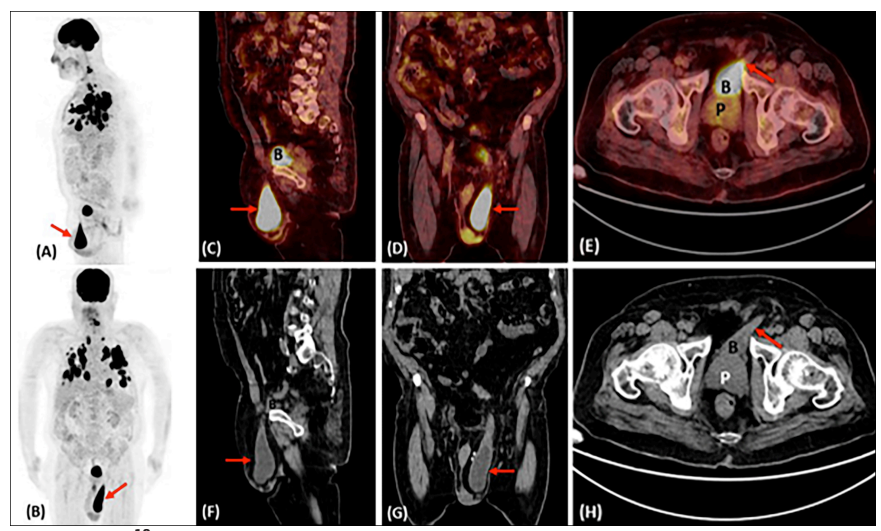

Figure 1: ${ }^{18} \mathrm{~F}$-fluorodeoxyglucose positron-emission tomography/computed tomography scan showing FDG uptake in the left-sided inguinoscrotal bladder hernia (red arrow). Sagittal and coronal maximum intensity projection images of whole-body (A-B). Sagittal, coronal, and axial fused positron-emission tomography/computed tomography images (CE). Sagittal, coronal, and axial computed tomography images (F-H). B, urinarybladder; P, prostate.

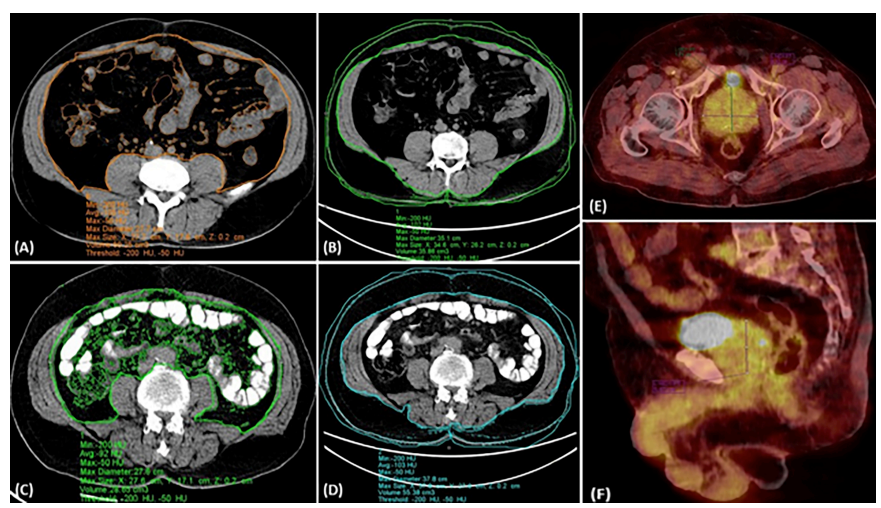

Figure 2: The measurement of abdominal visceral (VFV) and subcutaneous (SFV) fat volumes in axial computed tomography images, in which VFV is higher than SFV (A-B) and SFV is higher than VFV (C-D). The measurement of width, length, and the height of the prostate in axial and sagittal fused positron-emission tomography images (E-F).

\section{METHODOLOGY}

After receiving approval from the Institutional Research Ethics Committee (2021/3010), the patients who underwent ${ }^{18}$ F-FDG
PET/CT imaging because of a suspicious tumor site were referred for recurrence were retrospectively evaluated for the study. A written informed consent was obtained for all PET/CT examinations. Medical records of 6,819 males aged $>18$ years in the Department of Nuclear Medicine Digital Archives from August 2010 to April 2021, were undertaken. Patients diagnosed with ISH (111 cases) were detected. ISBH was observed in fifteen male patients $\geq 55$ years of age. Among 76 males with ISH of bowel, an age-matched group including fifteen males over 55 years was selected. Moreover, for comparison of measured parameters age-matched control group including 30 males without inguinal hernia was constructed. So, 60 patients were involved and subclassified in two groups; Group I with an ISH (30 cases) and Group II without inguinal hernia (30 cases). Group I was divided into two subgroups; Group la was composed of 15 patients with ISBH (Figure 1), and Group Ib consisted of $15 \mathrm{ISH}$ with bowel. Exclusion criteria were imaging files with low-resolution or incomplete visualization, patients with surgery for the prostate gland or inguinal hernia, and any evidence of the bladder tumor ormetastasis.

All images were acquired with an integrated ${ }^{18} \mathrm{~F}-\mathrm{FDG}$ PET/CT scanner (Siemens, Biograph 6 HiRes PET/CT, Germany) after the ${ }^{18}$ F-FDG injection. All patients fasted for at least 6 hours, and their blood glucose levels were $<200 \mathrm{mg} / \mathrm{dL}$. PET/CT imaging was performed from the skull base or vertex to the proximal thigh. First, CT imaging ( $130 \mathrm{kV}, 50 \mathrm{~mA}, 70-\mathrm{cm}$ field of view, and 2-mm section thickness) was performed without an IV contrast agent. Then, in three-dimensional acquisition mode, PET images were obtained with a 3-minute acquisition time for each bed. The iterative method was used to reconstruct PET images. One experienced radiologist and nuclear medicine physician interpreted PET/CT scans, and any differences of the metric variables were averaged.

The age, height, and weight of patients were obtained from the oncology department, and body mass index $\left(\mathrm{BMI}=\mathrm{Kg} / \mathrm{m}^{2}\right)$ was calculated by dividing weight $(\mathrm{Kg})$ by square of height $\left(\mathrm{m}^{2}\right)$. For analyzing PET/CT scans of Group la, in which there was a teardrop-shaped protruding hernial part of the bladder, primarily SUV $_{\max }$ values of intense ${ }^{18} \mathrm{~F}-\mathrm{FDG}$ accumulation in the hernia sac and urinary bladder, which are entirely separate two foci, were measured in sagittal fused PET images. Then, CT images were reviewed to measure other parameters, in which the size of the hernia sac, the volumes of the herniated and nonherniated parts of the bladder, prostatic tissue, abdominal subcutaneous, and visceral fat were measured using axial, coronal, and sagittal planes of CT images. The abdominal adipose tissue volumes were quantified by $2-\mathrm{mm}$ three slice CT scan at the L4 vertebral level. Three axial slices were acquired to measure the abdominal subcutaneous fat volume (SFV) and visceral fat volume (VFV) by measuring the mean value of all pixels within the range of -200 to -50 Hounsfield units. In these axial images, the VOI of the VFV was delineated between the inner margin of the abdominal wall muscles and the anterior border of the spinal column, whereas SFV was determined 
between the abdominal skin contour and the outer margin of the abdominal wall muscles (Figure 2). Then, the ratio of SFV to VFV was calculated and used to represent abdominal fat distribution. The mean volume of the prostate (PV) was calculated using the prolate ellipsoid formula; $\mathrm{PV}=$ width $\times$ length xheight $\times \pi / 6$ (Figure 2). ${ }^{12}$

Statistical analysis was performed using IBM SPSS (Statistical Package for the Social Sciences, version 25). Numerical data were expressed as mean \pm standard deviation, whereas categorical variables were presented as frequencies with percentages. Student t-test was used to compare the differences between the two groups. Correlation between continuous variables was analyzed by the Pearson correlation coefficient. Statistical significance was considered if $p<0.05$.

\section{RESULTS}

Of the 6,819 PET/CT scans of male patients screened, ISH with bowel and ISBH were observed with a frequency of $68.5 \%$ (76/111) and 13.5\% (15/111), respectively. In Group la, fifteen ISBH cases with the mean age of $72.07 \pm 10.51$ (55-90) years were included, and its frequency was $0.22 \%$. Left-sided ISBH was found in three patients (20\%), whereas in twelve patients, ISBH was right-sided (80\%). The clinical and demographic characteristics of cases with ISBH were summarised in Table I. The mean size of the hernia sac, hernia volume, and SUV ${ }_{\max }$ in a tear-drop shaped herniated and non-herniated bladder were found as $51 \pm 27 \mathrm{~mm}, 23.8 \pm 7.9 \mathrm{~mm}^{3}, 26.6 \pm 8.4$, and $37.3 \pm 9.8$, respectively. All ISBH cases withfocal intense ${ }^{18} \mathrm{~F}$-FDG uptake were classified as the extraperitoneal, in which the bladder herniated into the inguinal canal without peritoneum in PET/CT images.

In Group Ib, 15 males with the mean age of $71.87 \pm 10.2$ (55-91) years presented ISH with bowel hernia, in which five cases were right-sided, left-sided in three, and bilateral in seven patients. The mean size of the hernia sac and hernia volume were measured as $104.1 \pm 57 \mathrm{~mm}$, and $247.3 \pm 67.5 \mathrm{~mm}^{3}$, respectively. Group II included 30 cases without inguinal hernia as a control group with the mean age of $71.8 \pm 10.1$ (54-87) years. Thus, 60 cases ( 30 with and 30 without inguinal hernia) with a median age of $71.91 \pm 9.97(55-90)$ years were evaluated in this current study, and there was a homogeneous age distribution among these groups $(p=0.997)$.

There was a statistically significant association between Group $\mathrm{la}$ and $\mathrm{lb}$ in the measurements of BMI, the volumes of prostatic, subcutaneous and visceral fat tissues (Table II). The mean BMI, PV, SFV, and VFV of Group la $\left(28.54 \pm 3.94 \mathrm{Kg} / \mathrm{m}^{2}\right.$ for BMI, $41.80 \pm$ $7.85 \mathrm{~mm}^{3}$ for PV, $114.72 \pm 39.01 \mathrm{~mm}^{3}$ for SFV, and $177.11 \pm$ $65.55 \mathrm{~mm}^{3}$ for VFV) were higher than the measurements of Group $\mathrm{lb}$ and a statistical difference was determined between two groups $(p=0.006, p=0.012, p=0.003$, and $p=0.008$, respectively). In addition, there was a statistically significant difference between the SVF/VFV of the two groups $(p=0.005)$. Although the mean SFV and VFV of Group la were higher than that of Group Ib, the SVF/VFV value was smaller in Group Ib than
Group la, which indirectly reflects worse abdominal fat distribution. There was no statistical difference between the BMI of Group I and II $(p=0.084)$. Moreover, no significant relationship was found between the mean SFV values of two groups $(p=0.139)$. Conversely, a statistical difference was determined between the two groupsinterms of PV, VFV, and SFV/VFV parameters ( $p=0.035, p<0.001$ and $p<0.001$, respectively). Group II had a significantly higher SVF/VFV value compared with Group I $(1,22 \pm 0.47$ and $0.64 \pm 0.26, p<0.001)$ (Table II). So, Group I had a better abdominal fat distribution. Pearson's correlation showed an excellent positive correlation between BMI and the volumes of abdominal subcutaneous and visceral fat $(r=0.641$, $p<0.001$, and $r=0.724, p<0.001$ ). The authors found a significant moderately positive correlation between PV and VFV $(r=0.429, p=0.001)$. There was no significant correlation between PV and SFV ( $r=0.179, p=0.170)$. The SFV/VFV value showed a significant negative correlation with PV and VFV, whereas there was no correlation between SFV/VFV value and BMI, respectively $(r=-0.333, p<0.009, r=-0.457, p<0.001$, and $r=-0.130, p=0.323$ ).

\section{DISCUSSION}

Bladder-containing ISH, termed scrotal cystocele, is extremely rareand mostly detected in elderly obese patients. In the systematic review by Branchu et al., 61 cases diagnosed with bladder hernia between September 2005 and April 2017 were reported. ${ }^{2,13}$ Furthermore, Komorowski et al. reported 2 cases of IBH among 464 cases of inguinal hernias $(0.43 \%)$; whereas, three hernia sac containing the bladder were reported in 54 patients with inguinal hernias $(5.5 \%)$ in a study by Patle et al. ${ }^{14,15}$ On review of the literature, approximately 8-10 case reports of IBH, which were incidentally detected on PET/CT imaging, could be identified. ${ }^{7,9-11,16-19}$ Previous studies reported that FDG uptake in the herniated bladder is an important pitfall that may be misinterpreted as tumor metastases. The possibility of misinterpreting the increased focal intense FDG activity in the inguinal canal can be prevented by means of having simultaneous coregistered CT images. ${ }^{7,9}$ One of the important consequences of this study was the higher prevalence of ISBH in PET/CT imaging, which suggested a possible role of the ability to evaluate the patient by scanning whole-body from the skull base to the midthigh. However, PET/CT imaging allows a more objective modality to determine the incidence of ISBH by detecting an intense ${ }^{18} \mathrm{~F}$-FDG accumulation in the herniated bladder, even if very small that may be overlooked on physical examination or not captured in other imaging modalities. In this retrospective study, the authors detected ISBH in 15 (3 left-sided and 12 right-sided) of 6,819 PET/CT scans of male patients $(0.22 \%)$; whereas, the incidence of $\mathrm{ISBH}$ among all inguinal hernias was found as $13.5 \%$. This high incidence may be explained by missed diagnosis of ISH due to lack of ${ }^{18} \mathrm{~F}-\mathrm{FDG}$ accumulation or hernia sac that is too small to detectin CT or MR imaging.

Unusual herniation of the urinary bladder into the inguinal canal may increase the risk of ischemia, perforation, incarceration or strangulation of the bladder. ${ }^{3,6,7,13}$ 
Table I: Summary of clinical characteristics of inguinoscrotal bladder hernia cases.

\begin{tabular}{|c|c|c|c|c|c|c|c|}
\hline Case & Age & Cancer history & Side & $\begin{array}{l}\text { HS } \\
(\mathrm{mm})\end{array}$ & $H V\left(\mathrm{~mm}^{3}\right)$ & $\begin{array}{l}\mathbf{H} \\
\text { SUV }_{\max }\end{array}$ & UB SUV $_{\text {max }}$ \\
\hline 1 & 67 & Lung & $\mathrm{R}$ & 30 & 4.29 & 20.14 & 18.44 \\
\hline 2 & 69 & Lung & $\mathrm{L}$ & 80 & 61.19 & 64.92 & 46.6 \\
\hline 3 & 69 & Lung & $\mathrm{R}$ & 50 & 20.15 & 13.83 & 17.13 \\
\hline 4 & 84 & Lung & $\mathrm{R}$ & 45 & 8.68 & 17.88 & 44.77 \\
\hline 5 & 85 & Lung & $\mathrm{R}$ & 35 & 4.84 & 63.50 & 24.22 \\
\hline 6 & 55 & Prostate & $\mathrm{R}$ & 40 & 6.26 & 3.61 & 13.45 \\
\hline 7 & 73 & Prostate & $\mathrm{R}$ & 65 & 57.47 & 22.30 & 11.76 \\
\hline 8 & 74 & Prostate & $\mathrm{R}$ & 40 & 8.06 & 19.64 & 24.61 \\
\hline 9 & 90 & Prostate & $\mathrm{R}$ & 20 & 2.33 & 21.43 & 47.34 \\
\hline 10 & 55 & $\mathrm{RCC}$ & $\mathrm{L}$ & 40 & 6.22 & 19.89 & 18.77 \\
\hline 11 & 61 & Pancreas & $\mathrm{R}$ & 20 & 1.09 & 32.60 & 103.38 \\
\hline 12 & 76 & Lymphoma & $\mathrm{R}$ & 70 & 57.41 & 40.30 & 32.87 \\
\hline 13 & 67 & Thyroid & $\mathrm{L}$ & 115 & 98.44 & 21.19 & 17.82 \\
\hline 14 & 83 & $\mathrm{KS}$ & $\mathrm{R}$ & 50 & 16.03 & 9.07 & 127.29 \\
\hline 15 & 73 & MM & $\mathrm{R}$ & 90 & 72.75 & 29.08 & 11.23 \\
\hline
\end{tabular}

Table II. Comparison of measured parameters between the control group and inguinoscrotal herniation groups (Group la and Ib).

\begin{tabular}{|c|c|c|c|c|c|c|}
\hline Parameters & Group la $(n=15)$ & Group Ib $(n=15)$ & p value & Group I $(n=30)$ & Group II $(n=30)$ & p value \\
\hline $\operatorname{BMI}\left(\mathrm{kg} / \mathrm{m}^{2}\right)$ & $28.54 \pm 3.94$ & $24.54 \pm 3.46$ & 0.006 & $26.54 \pm 3.78$ & $24.94 \pm 3.46$ & 0.084 \\
\hline $\mathrm{PV}\left(\mathrm{mm}^{3}\right)$ & $41.80 \pm 7.85$ & $33.59 \pm 8.84$ & 0.012 & $37.70 \pm 9.21$ & $30.75 \pm 15.06$ & 0.035 \\
\hline SFV $\left(\mathrm{mm}^{3}\right)$ & $114.72 \pm 39.01$ & $67.40 \pm 36.23$ & 0.003 & $91.06 \pm 38.35$ & $108.31 \pm 43.60$ & 0.139 \\
\hline $\operatorname{VFV}\left(\mathrm{mm}^{3}\right)$ & $177.11 \pm 65.55$ & $113.92 \pm 54.57$ & 0.008 & $145.51 \pm 59.63$ & $94.07 \pm 41.76$ & 0.001 \\
\hline SFV/VFV & $0.69 \pm 0.25$ & $0.59 \pm 0.26$ & 0.005 & $0.64 \pm 0.25$ & $1.22 \pm 0.47$ & 0.001 \\
\hline
\end{tabular}

Most of the inguinal bladder hernias are diagnosed intraoperatively $(77 \%)$; whereas, $7 \%$ of them are detected incidentally during preoperative radiologic imaging. Moreover, iatrogenic injury of the bladder during surgical interventions can develop inguinal bladder hernia postoperatively (16\%)., ${ }^{5,8,13}$ Preoperative diagnosis of ISBH at an early stage is crucial to prevent iatrogenic bladder injuries and other severe complications. Awareness of the underlying causes or risk factors facilitates predicting and preventing the progression and recurrence of $\mathrm{ISBH}{ }^{5,7,10}$

Multiple factors such as obesity, BPH, increasing intra-abdominal pressure, abdominal wall weakness or defect, decreased bladder tone, or bladder outlet obstruction play a role in ISBH etiopathogenesis. ${ }^{5,11,13}$ The impact of major risk factors on ISBH development has not been evaluated in previous studies. To the authors' knowledge, this is the first retrospective study that examines the risk factors associated with the development of ISBH using current PET/CT imaging. Conversely, in this study, some of the cases with ISBH had normal or decreased PV; and the average BMI was $28.54 \pm 3.94 \mathrm{Kg} / \mathrm{m}^{2}$ (range from 22 to 37 ), which was not excessively high as VFV value. Although this may seem contradictory, the relationship between the PV; VFV, and BMI in Group la may be related to weight loss in cancer patients or previous prostate resection. Tumor cachexia can cause a decrease in the volume of abdominal fat tissues. To overcome this problem, the authors evaluated PET/CT images with the lowest tumor burden among the patient' sequential $\mathrm{PET} / \mathrm{CT}$ scans.
Several studies have indicated that abdominal obesity (lower SFV/VFV) associated with increased intra-abdominal pressure, may result in ISH development. ${ }^{2,5,13}$ In this study, BMI, $\mathrm{PV}, \mathrm{SVF}$, and VFV were compared by the content of the ISH sac and a statistically significant difference was found between Group la and Ib $(p<0.05)$. However, there was no significant difference between Group I and II in terms of BMI and SFV ( $p>0.05)$. In addition, PV was the most remarkable factor affecting the development of ISBH in this study; whereas, lower SFV/VFV was a more prominent factor that increased the risk of ISH with bowel when the authors compared the patients in subgroups of Group I. There was a significant positive correlation between $\mathrm{BMI}$ and the volumes of abdominal subcutaneous and visceral fat.

Limitations of this study include a retrospective design from a single centre with a relatively small sample size. Due to the retrospective design, factors suggesting bladder outlet obstruction and thus contributing to the risk of hernia formation, such as intravesical prostatic protrusion (IPP) and postvoid residual volume, could not be evaluated. Moreover, there is no information on the differences between Group la and $\mathrm{Ib}$ in terms of clinical symptoms and prognosis. However, this study represents the first series that compares rare interesting cases of ISBH with ISH with bowel in relation to major risk factors. In addition, having a control group is a positive aspect of this study. Nevertheless, further large-scale, multicentric retrospective studies are needed to confirm the efficacy of major risk factors on the development of ISBH. 


\section{CONCLUSIONS}

There was a relatively higher frequency of incidental detection of ISBH on ${ }^{18} \mathrm{~F}-\mathrm{FDG}$ PET/CT scans. A higher PV had a significant association for the development of ISBH as well as lower SFV/VFV that was more prominent in ISH with bowel. Incidental $\mathrm{PET} / \mathrm{CT}$ detection of ISBH is of interest in the correct interpretation of radiologic images, planning of modified surgical approaches, and preventing iatrogenic injury.

\section{ETHICAL APPROVAL:}

This retrospective observational study was performed at the Department of Nuclear Medicine, Meram Faculty of Medicine, Necmettin Erbakan University, Konya, Turkey. Local Ethics Committee approval (date:08/01/2021; approval number: 2021/3010).

\section{PATIENTS' CONSENT:}

A written informed consent was obtained from all patients included in the study.

\section{CONFLICT OF INTEREST:}

The authors declared no conflict of interest.

\section{AUTHORS' CONTRIBUTION:}

GA: Conception and design, analysis and interpretation of data, drafting of the manuscript, advices and final approval.

OS: Analysis and interpretation of data, conception and design, reviewed the paper, and final approval.

\section{REFERENCES}

1. Hernia Surge Group. International guidelines for groin hernia management. Hernia 2018; 22(1):1-165. doi: 10.1007/s10029-017-1668-x.

2. Branchu B, Renard Y, Larre S, Leon P. Diagnosis and treatment of inguinal hernia of the bladder: A systematic review of the past 10 years. Turk J Urol 2018; 44(5):384-8. doi: 10.5152/tud.2018.46417.

3. Bisharat M, O'Donnell ME, Thompson T, MacKenzie N, Kirkpatrick D, Spence RA, et al. Complications of inguinoscrotal bladder hernias: a case series. Hernia 2009; 13(1):81-4. doi: 10.1007/s10029-008-0389-6.

4. Foladi N, Farzam F, Tahir Aien M. Massive inguino-scrotal herniation of urinary bladder in an infant (scrotal cystocele)-case report. Radiol Case Rep 2020; 15(5):607-9. doi: 10.1016/j.radcr.2020.02.024.

5. Kaya T, Atici SD. Urinary bladder herniation in the differential diagnosis of inguinal hernia. Med Sci 2021; 10:132-5. doi: 10.5455/medscience.2020.08.167.

6. Erbin A, Canat HL, Ucpinar B, Malcik H. Bladder Hernia, Bladder Stone and Contralateral Bowel Hernia: An Usual Combination. J Coll Physicians Surg Pak 2019; 29(6):S62-4. doi: 10.29271/jcpsp.2019.06.S62.

7. Vadi SK, Mittal BR, Singh H, Kumar R, Dhiman RK. Incidental detection of urinary bladder herniation in 18F-fluorodeoxyglucose positron emission tomography/computed tomography mimicking as metastatic deposit in the Inguinal canal. Indian J Nucl Med 2019; 34(3):247-8. doi: 10.4103/ijnm.IJNM_38_19.

8. Wang $P$, Huang $Y$, Ye J, Gao G, Zhang F, Wu H. Large sliding inguino-scrotal hernia of the urinary bladder. Medicine (Baltimore) 2018; 97(13):e9998. doi: 10.1097/MD. 0000000 000009998.

9. Park SH, Kim MJ, Kim JH, Cho AE, Park MS, Kim KW. FDG uptake in PET by bladder hernia simulating inguinal metastasis. Yonsei Med J 2007; 48(5):886-90. doi: 10.3349/ ymj.2007.48.5.886.

10. Gupta N, Verma R, Belho ES. Herniated urinary bladder detected on F-fluorodeoxglucose positron-emission tomography/computed tomography scan imitating as Ffluorodeoxyglucose avid lesion. Indian I Nucl Med 2020; 35(2):172-3. doi: 10.4103/ijnm.JJNM_7_20.

11. Erkan ME, Yılmaz S, Kaya ZR, Sager S, Tan YZ, Halaç M. Inguinal herniation of the bladder mimicking malignancy in FDG PET/CT: Original Image. Turkiye Klinikleri J Med Sci 2012; 32(3):895-7. doi: 10.5336/medsci.2011-24353.

12. Zlotta AR, Djavan B, Damoun $M$, Roumeguere $T$, Petein $M$, Entezari K, et al. The importance of measuring the prostatic transition zone: An anatomical and radiological study. BJU Int 1999; 84(6):661-666. doi: 10.1046/j.1464-410x. 1999.00214.x.

13. Gonzalez-Urquijo M, Mayagoitia-Gonzalez JC, Rodarte-Shade M, Bermea-Mendoza JH, Gil-Galindo G. Large inguinal bladder hernias: Can a preoperative diagnosis be made? Hernia 2019; 23(6):1221-7. doi: 10.1007/s10029019-01955-3.

14. Komorowski AL, Moran-Rodriguez J, Kazi R, Wysocki WM. Sliding inguinal hernias. Int J Surg 2012; 10(4):206-8. doi: 10.1016/j.ijsu.2012.03.002.

15. Patle NM, Tantia O, Prasad P, Khanna S, Sen B. Sliding inguinal hernias: Scope of laparoscopic repair. J Laparoendosc Adv Surg Tech A 2011; 21(3):227-31. doi: 10.1089/lap.2010.0473.

16. Shahlaei S, Shakeri S, Farahmandfar F, Emami F, Sadeghi R. Inguino-scrotal bladder hernia: An unexpected finding on 68Ga-PSMA-11 PET/CT. Asia Ocean J Nucl Med Biol 2020; 8(2):141-4. doi: 10.22038/AOJNMB.2020.45247.1305.

17. Usmani S, Marafi F, Ahmed N, Kazem N. Inguinal herniation of urinary bladder on F-18 sodium fluoride (NaF) PET-CT. Nucl Med Mol Imaging 2017; 51(4):368-70. doi: 10.1007/s13139-016-0431-z.

18. Hinojosa $D$, Joseph UA, Wan DQ, Barron BJ. Inguinal herniation of a bladder diverticulum on PET/CT and associated complications. Clin Imaging 2008; 32(6):483-6. doi: 10.1016/j.clinimag.2008.03.003.

19. Harisankar CN. Incidentally detected vesico inguinal hernia on fluorodeoxy glucose positron emission tomography-computed tomography. Indian J Nucl Med 2013; 28(2):127-8. doi: 10.4103/0972-3919.118262. 